\title{
Necessity of Studying the Methods for Isolation of Nocardia from Environmental and Clinical Samples
}

\author{
Masoud Keikha1,* \\ ${ }^{1}$ Department of Microbiology, School of Medicine, Isfahan University of Medical Sciences, Isfahan, IR Iran \\ "Corresponding author: Masoud Keikha, Department of Microbiology, School of Medicine, Isfahan University of Medical Sciences, Isfahan, IR Iran. Tel: +98-9386836425, E-mail: \\ masoudkeikha@outlook.com
}

Received 2017 July 15; Revised 2017 August 12; Accepted 2017 October 14.

\section{Dear Editor,}

Nocardia is a genus of gram-positive, aerobic, slow growth, and partial acid fast bacteria. It is free-living species (saprophytic) existing in environmental resources such as water, soil, dust, animal waste and rotten plants. Therefore, the patients with immunodeficiency have a high chance of getting infections with these bacteria. According to available evidence, Nocardia species enter the human body through dust, ventilation systems and traumatic inoculations and cause Noccardial infections in patients with immunodeficiency and the healthy individuals $[1,2]$.

Nocardial infections often occur as Nocardial pneumonia infections, brain abscesses, cutaneous, lymphatic, and lympho-cutaneous infections. The isolation of Nocardia species is of high significance due to the clinical importance and the production of secondary metabolites, especially antibiotics [1-4]. However, it should be noted that it is difficult to isolate Nocardia species because of the complexities involved in isolating and identifying this group of bacteria. The clinical manifestations and radiological evidence of nocardial pulmonary infections are nonspecific and the diagnosis of nocardiosis based on the clinical findings is impossible. Also, the Nocardia species are slowly growing, thus, other fast-growing bacteria prevent the isolation of these bacteria. Also diagnosis of nocardiosis based on direct examination and conventional cultures, is difficult, long, time-consuming and given to time-consuming conventional tests, nocardiosis is sometimes diagnosed after the dissemination of the strain to other organs or after the patient has died; so we need for new methods for identification of Nocardia infections. The molecular methods such as $16 \mathrm{~S}$ rRNA-restriction fragment length polymorphism (16S rRNA-RFLP), hsp65-RFLP, direct sequencing of 16S rRNA and hsp65 was eliminates these problems by the specific amplification of Nocardia genome even after the beginning of chemotherapy $[3,5]$.

Different confirmed scientific methods have been in- troduced to isolate these bacteria from clinical and environmental samples, including: Paraffin agar, Paraffin baiting technique, Humic acid vitamin B agar, Selective BCYE (Buffered charchol-yeast exctract), Slip-buried method, sucrose-gradient centrifugation, gelatin agar (GA), Blood agar and urea agar [3]. Among the methods mentioned above, the Paraffin baiting technique is one of the most important methods for the isolation of Nocardia species from clinical and environmental samples which is widely used. Nocardia species having paraffin degrading enzymes are able to use this compound as the only source of carbon and energy. Therefore, one can isolate Nocardia species from polymicrobial samples such as soil or sputum easily using an organic-free medium (for example McClung medium) and adding paraffin rods to it. Although it has been shown that some Pseudomonas species can also use paraffin, this method has been used in several studies on the isolation of Nocardia species from clinical and soil samples and has the advantage of isolating more Nocardia species compared to other methods [6-9]. According to Hayakawa et al. other genera of Streptomyces, Micromonospora, Microbispora, Streptosporangium, Dactylosporangium, Microtetraspora and Thermomonospora are also isolated by Humic acidvitamin B agar technique in addition to Nocardia. Therefore, HV agar is not a specific environment for Nocardia species [3]. Eshraqi et al. found that paraffin agar is not a specific method for the isolation of Nocardia species due to contamination with other microorganisms [3]. The Slipburied technique is not also a specific method for the isolation of Nocardia species due to the use of Streptomycin and Chloramphenicol antibiotics and the sensitivity of some Nocardia species to these antibiotics [10]. It is difficult and time consuming to isolate Nocardia species using phenotypic methods and it is suggested to use molecular methods such as hybridization or examination of 16S rRNA and hsp65 genes in urgent cases and need for immediate diagnosis of Nocardia infections. Nevertheless, it should be noted that phenotypic methods are known as gold stan- 
dard, and molecular methods are complementary to these methods. Also, molecular techniques' equipment are expensive and require trained technicians. Thus, it is not possible to use molecular techniques in all medical diagnostic laboratories in developing countries $[4,11]$.

In summary, isolation and identification of Nocardia species are important due to their clinical and industrial application and studies on the identification of new species of Nocardia. According to the research, it can be stated that the Paraffin baiting technique is one of the most reliable methods for the isolation of Nocardia species from clinical and soil samples. In addition, it is likely in clinical cases that Nocardia attacks other organs of the patient body and causes the patient' death prior to phenotypic identification. Therefore, in such cases, molecular methods can be used.

\section{Footnote}

Conflict of Interest: There is no conflict of interest.

\section{References}

1. Brown-Elliott BA, Brown JM, Conville PS, Wallace RJ. Clinical and laboratory features of the Nocardia spp. based on current molecular taxonomy. Clin Microbiol Rev. 2006;19(2):259-82. doi: 10.1128/CMR.19.2.259-282.2006. [PubMed: 16614249].
2. Kageyama A, Yazawa K, Kudo T, Taniguchi H, Nishimura K, Mikami Y. First isolates of Nocardia abscessus from humans and soil in Japan. Nihon Ishinkin Gakkai Zasshi. 2004;45(1):17-21. [PubMed: 14765097].

3. Rasouli-Nasab M, Fatahi-Bafghi M, Habibnia S, Heidarieh P, Eshraghi SS. Comparison of Various Methods for Isolation of Nocardia from Soil. Zahedan J Res Med Sci. 2017;In Press(In Press) doi: 10.5812/zjrms.6107.

4. Fatahi Bafghi M, Saeed Eshraghi S, Heidarieh P, Habibnia S, Nasab MR. Nocardiosis in immune disorder disease. Malaysian J Med Sci. 2014;21(1):75-6.

5. Couble A, Rodriguez-Nava V, de Montclos MP, Boiron P, Laurent F. Direct detection of Nocardia spp. in clinical samples by a rapid molecular method. J Clin Microbiol. 2005;43(4):1921-4. doi: 10.1128/JCM.43.4.1921-1924.2005. [PubMed: 15815019].

6. Mishra SK, Randhawa HS. Application of paraffin bait technique to the isolation of Nocardia asteroides from clinical specimens. Appl Microbiol. 1969;18(4):686-7. [PubMed: 4905040].

7. Narang $P$, Narang R, Bhattacharya S, Mendiratta DK. Paraffin slide culture technique for isolating non-tuberculous mycobacteria from stool and sputum of HIV sero-positive patients. Indian J Tuberc. 2004;1(1):23-6.

8. Patel DD, Lakshmi B. Study on the role ofNocardia farcinicain enhancing the flow rate of crude oil. Bioremediat J. 2016;20(3):224-32. doi: 10.1080/10889868.2016.1212806.

9. Bafghi MF, Heidarieh P, Soori T, Saber S, Meysamie A, Gheitoli K, et al. Nocardia isolation from clinical samples with the paraffin baiting technique. Germs. 2015;5(1):12-6. doi: 10.11599/germs.2015.1066. [PubMed: 25763363].

10. Bafghi MF, Eshraghi SS. Nocardia Isolation of Soil. Adv Biomed Res. 2017;6:1. doi: 10.4103/2277-9175.199262. [PubMed: 28217646].

11. Singh A, Goering RV, Simjee S, Foley SL, Zervos MJ. Application of molecular techniques to the study of hospital infection. Clin Microbiol Rev. 2006;19(3):512-30. doi: 10.1128/CMR.00025-05. [PubMed: 16847083]. 